implementation of the ERAS protocol and adherence to all ERAS items contribute to better outcomes in gynecological oncology surgery.

The aim of this survey is to acquire a comprehensive picture of the current status of implementation of the ERAS protocol among European centers.

Methodology A 45-item questionnaire survey, investigating ERAS practice preferences in pre-, intra-, and post-operative management was launched between December 2020 and May 2021. An on-line questionnaire link was e-mailed to the national representatives (NATREPs) of the European Network of Young gynecologic oncologists (ENYGO), who then shared the survey with their centers. In order to avoid duplicate data, NATREPs selected one referral person from the highest volume centers performing complex surgery per country. Two weeks later a reminder was sent to non-responders.

Result(s)* A total of 116 responses were collected. $73 \%$ of centers were academic/teaching hospitals. Overall, 70\% of respondents reported that ERAS was implemented at their institution. The median LOS for advanced ovarian cancer surgery was between 5-7 days according to 63\% of respondents. $81 \%$ of respondents reported a median LOS between 2-4 days among patients who underwent surgery for early-stage gynecological cancer. An overall compliance rate between $60 \%$ and $80 \%$ was reported by $44,29 \%$ of centers.

ERAS items with well adherence to the guidelines were: deep vein thrombosis prophylaxis, antibiotic prophylaxis, prevention of hypothermia and early mobilization. Regarding preoperative ERAS items, 28\% of respondents reported bowel preparation as 'sometimes-normally' performed. $60 \%$ of respondents described ERAS implementation as a challenge and $76 \%$ reported being reluctant to change clinical practice.

Conclusion* This European survey of ERAS in gynecologic oncology surgery shows that there is still a lack of implementation of the ERAS protocol across Europe and European centers need to increase ERAS gynecologic oncology guideline compliance to improve patient outcomes. Therefore, European centers need a further protocol and guidelines that encompass the way of ERAS implementation

\section{THE LOCKDOWN EFFECT ON GYNAECOLOGICAL CANCER SURGERIES DURING THE COVID-19 PANDEMIC}

V Theodoulidis*, DE Vlachos, C Theofanakis, V Pergialiotis, N Thomakos, A Rodolakis, D Haidopoulos. National University of Athens, First Department of Obstetrics and Gynecology , Athens, Greece

\subsection{6/ijgc-2021-ESG0.318}

Introduction/Background* The impact of COVID-19 pandemic caused a disruption of the healthcare systems and led to significant delays in diagnosis and treatment of gynecological cancer patients. New algorithms that aim to sustain balance between management of oncological patients and the need to maintain a sufficient amount of resources were adapted.

Methodology This retrospective study reviewed the patients with gynecological cancer operated in our hospital during the first lockdown period in Greece (between 13 March 2020 and 30 May 2020) and compared the results with the corresponding time period in 2019 before COVID-19 pandemic. We also examine the number of patients that were referred for neoadjuvant chemotherapy or radiotherapy between those periods. Result(s)* The gynecological oncological operations performed during the lockdown period of the first pandemic wave were not altered by the outbreak (153 in 2019 vs 130 in 2020) (figure 1). There was no difference in ovarian cancer surgeries (34 vs 31) and the number of primary debulking was not affected (20 vs 19). The patients referred to neoadjuvant chemotherapy was the same between the two periods. No significant difference was obtained in the endometrial, vaginal and cervical cancer surgeries and the number of surgically treated recurrences. Contrastingly there was a significant decrease in endoscopic procedures and diagnostic biopsies (72 vs 53) and the fertility sparring surgeries were postponed from patients at a later date (table 1).

Conclusion* The findings of our study suggest that at the first wave of the pandemic lockdown, the operations conducted in our department did not alter. In accordance with international consensus guidelines the stage, the grade and the type of cancer, and the potential comorbidities were the main factors that accounted for the decision of the optimal mode of treatment.

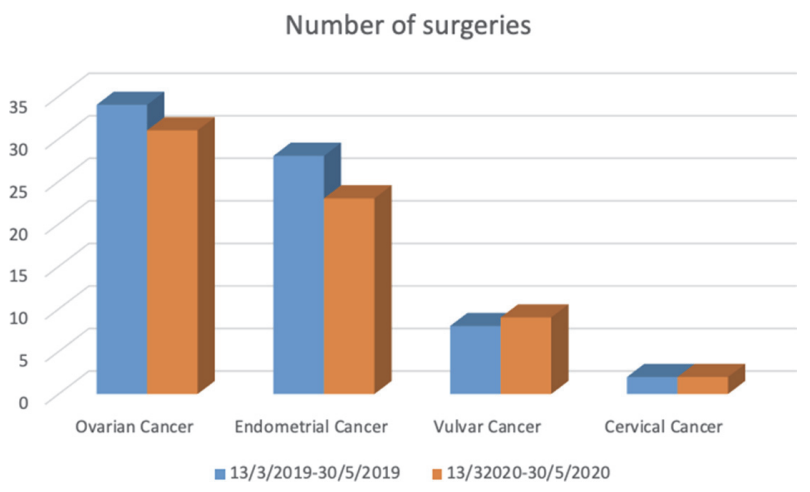

Abstract 798 Figure 1 Gynecological cancer surgeries on both periods

$\begin{array}{lcc}\text { Abstract } 798 \text { Table } 1 & \text { Type of procedures on both periods } \\ \text { OVARIAN CANCER } & \mathbf{1 3 / 3 / 2 0 1 9}-\mathbf{3 0 / 0 5 / 2 0 1 9} & \mathbf{1 3 / 3 / 2 0 2 0}-\mathbf{3 0 / 5 / 2 0 2 0} \\ \text { Primary Cytoreduction } & 34 & \mathbf{3 1} \\ \text { Interval - Late Debulking } & 20 & 19 \\ \text { Neoadjuvant Chemotherapy } & 5 & 6 \\ \text { Final Benign Histology } & 7 & 7 \\ \text { ENDOMETRIAL CANCER } & 9 & 6 \\ \text { CERVICAL CANCER } & \mathbf{2 8} & \mathbf{2 3} \\ \text { Radical Hysterectomy } & \mathbf{2} & \mathbf{2} \\ \text { Trachylectomy } & 1 & 2 \\ \text { RECURRENCE } & 1 & - \\ \text { Ovarian Cancer } & 7 & 7 \\ \text { Endometrial Cancer } & 3 & 3 \\ \text { Cervical Cancer } & 2 & - \\ \text { Vulvar Cancer } & - & 1 \\ \text { REOPERATION } & 2 & 3 \\ \text { DIAGNOSTIC LAPAROSCOPY } & \mathbf{2} & \mathbf{5} \\ \text { MINOR PROCEDURE } & \mathbf{1 1} & \mathbf{8} \\ \text { Pigtail - Cystoscopy } & 61 & 45 \\ \text { EUA - Biopsy - D\&G } & 42 & 31 \\ \text { FERTILITY SPARRING SURGERY } & 19 & 14 \\ \end{array}$

